\title{
Sports performance research under the spotlight
}

\author{
G. Atkinson ${ }^{1}$, A.M. Batterham ${ }^{1}$ and W.G. Hopkins ${ }^{2}$ \\ ${ }^{1}$ Health and Social Care Institute, Teesside University, Middlesbrough, \\ UK
${ }^{2}$ Institute of Sport and Recreation Research, AUT University, Auckland, New Zealand

In July 2012 a three-pronged "attack" on the quality of sports performance research was launched in the UK. First, a major report from a House of Lords inquiry was published (4). One of the many unsettling conclusions was that,

"....there is little evidence to suggest that the enhancement of the performance of elite athletes is generally based on strong biomedical science, nor that the latest advances in relevant areas of biomedical research are consistently applied to this work."

Second, the British Medical Journal published a series of articles and commentaries that were highly critical of research on the ergogenic effects of sports drinks. The leading review article was entitled "Forty years of sports performance research and little insight gained" (2). Third, the widespread criticisms in this review were conveyed in the BBC's popular investigative programme "Panorama". Expensive running shoes were also depicted as potentially harmful in the television programme.

We have concerns about these reports and the BBC programme. However, we suspect that a review of any scientific discipline would find the same mix of good, bad and ugly research. Therefore, these critical reports should serve as reminders to authors that IJSM sets high benchmarks for the rationale, design and analysis of published studies. Alongside the usual IJSM submission guidelines, we advise all potential authors of the following important good practice points;

1. A relevant population should be selected in tandem with the particular research question. The study population should be clearly defined as Olympic athletes, recreational athletes, etc., rather than using ambiguous terms such as "elite" or "sub-elite". Athletic categories from sports governing bodies and/or world rankings of participants should be stated, where possible.

2. Performance outcomes should be directly relevant to the study population. Correlational studies identifying predictors of performance are justifiable only if it is difficult to measure or simulate (e.g. via a time trial) the athletic 
performance directly. Team sports need careful consideration in this respect. One should ask oneself, "Why do I want to predict sports performance when it may be already directly measurable?"

3. Statistical significance and non-significance can no longer be taken as sole evidence for the presence or absence of a clinically/practically meaningful effect. Authors must present the precision of any sample-based estimate with confidence intervals, and these should be interpreted alongside a wellrationalised minimal worthwhile effect. Authors are advised to consult the relevant guidelines published recently in Med Sci Sports Exerc (3).

4. There should be no speculation about the value to athletes based purely on biological outcomes, e.g., heart-rate responses, unless the relationship between biological outcome and performance outcome is clear and strong. A past editorial should be consulted so that a particular study can be placed within the framework performance enhancement research (1). The difference between efficacy and effectiveness of an intervention should be appreciated.

5. Individual responses to any treatment should be described by the standard deviation of change data. Graphical presentation of individual data is encouraged. Athlete characteristics (e.g., gender, age, level of training or competition) that could account for individual responses should also be considered and, where possible, included as moderators in the data analysis.

6. Any strict control of diet or physical activity should be clearly rationalised. Athletes in the real world do not fast for $12 \mathrm{~h}$ prior to an event.

\section{References}

1. Atkinson $G$, Batterham AM, Drust B. Is it time for sports performance researchers to adopt a clinical-type research framework? Int J Sports Med 2008; 29: 703-705.

2. Heneghan C, Perera R, Nunan D, Mahtani K, Gill P. Forty years of sports performance research and little insight gained. Brit Med J 2012; 345: e4797

3. Hopkins WG, Marshall SW, Batterham AM, Hanin J. Progressive Statistics for Studies in Sports Medicine and Exercise Science. Med Sci Sports Exerc 2009; 41: 3-12.

4. House of Lords Select Committee on Science and Technology. Sport and exercise science and medicine: building on the Olympic legacy to improve the nation's health. London, The Stationary Office Limited, 2012: 4, Available at: http://www.publications. parliament.uk/pa/ld201213/ldselect/ldsctech/33/33.pdf 